\title{
The silencing of FNDC1 inhibits the tumorigenesis of breast cancer cells via modulation of the PI3K/Akt signaling pathway
}

\author{
CHEN YUNWEN $^{1,2}$, GAO SHANSHAN $^{1}$, BEN ZHIFEI $^{1}$, CHEN SAIJUN $^{1}$ and YIN HUA $^{1}$ \\ ${ }^{1}$ Hwa Mei Hospital, and ${ }^{2}$ Ningbo Institute of Life and Health Industry, \\ University of Chinese Academy of Sciences, Ningbo, Zhejiang 315010, P.R. China
}

Received May 25, 2020; Accepted September 28, 2020

DOI: $10.3892 / \mathrm{mmr} .2021 .12118$

\begin{abstract}
Fibronectin type III domain-containing protein 1 (FNDC1) is a protein that contains a major component of the structural domain of fibronectin. Although many studies have indicated that FNDC1 serves vital roles in the development of various diseases, the role of FNDC1 in the progression of breast cancer $(\mathrm{BC})$ remains elusive. The aim of the present study was to investigate the biological functions of FNDC1 in $\mathrm{BC}$ cells and the associated mechanisms. The expression levels of FNDC1 in BC tissues and normal breast tissues were analyzed using The Cancer Genome Atlas database (TCGA). Kaplan-Meier curves were mined from TCGA to examine the clinical prognostic significance of FNDC1 mRNA in patients with BC. The expression of FNDC1 was knocked down by transfection with shRNA in BC cells. Cell viability, colony formation ability, migration and invasion were assayed following the silencing of FNDC1 in BC cells. The expression of proteins was measured using a western blotting assay. The bioinformatic data indicated that the FNDC1 mRNA expression levels were significantly upregulated in $\mathrm{BC}$ tissues compared with normal breast tissues, and the high mRNA expression levels of FNDC1 were associated with a lower overall survival in patients with BC. The downregulation of FNDC1 inhibited the proliferation, colony formation, migration and invasion of $\mathrm{BC}$ cells. Investigation of the mechanisms revealed that the silencing of FNDC1 decreased the protein expression levels of MMPs and epithelial-to-mesenchymal markers. Furthermore, the silencing of FNDC1 led to the inactivation of the PI3K/Akt signaling pathway. FNDC1 was highly upregulated and acted as an oncogene in BC. Therefore, targeting FNDC1 may be a potential strategy for the treatment of $\mathrm{BC}$.
\end{abstract}

Correspondence to: Dr Yin Hua, Hwa Mei Hospital, University of Chinese Academy of Sciences, 41 Northwest Street, Ningbo, Zhejiang 315010, P.R. China

E-mail: yhnb320@163.com

Key words: breast cancer, fibronectin type III domain-containing protein 1, tumorigenesis, PI3K/Akt

\section{Introduction}

Breast cancer (BC) is one of the most commonly diagnosed cancer types among females worldwide (1). Although great efforts have been made in the treatment of $\mathrm{BC}$, the overall survival of patients with $\mathrm{BC}$ remains poor (2). Local tumor invasion and distant metastasis are key reasons that account for the poor survival of patients with BC in the advanced stages (3). Therefore, it is necessary to reveal the underlying mechanisms of progression of BC to develop more effective therapeutic targets.

The fibronectin type III domain-containing protein 1 (FNDC1), also known as AGS8, contains the conserved fibronectin type III domain of fibronectin (FN1) (4). FN1 was documented as an essential player in tumorigenesis and has been shown to affect various physiological processes, including proliferation, migration, metabolism and apoptosis (5).

It has been reported that the upregulation of intracellular FN1 is associated with distant metastasis of BC (6). Numerous studies have demonstrated that FNDC1 also serves critical roles in different diseases. For instance, the upregulation of FNDC1 has been associated with skin tumor progression and increases in tumor thickness (7). In prostate cancer cells, the silencing of FNDC1 inhibited proliferation and migration while increasing apoptosis (8). FNDC1 was also found to be highly expressed in gastric cancer, and the upregulation of FNDC1 was associated with a poor prognosis in patients with gastric cancer. However, the role of FNDC1 in $\mathrm{BC}$ has not been studied yet.

Therefore, in the present study, The Cancer Genome Atlas (TCGA) database was used to compare the mRNA expression levels of FNDC1 in BC and normal breast tissues. The Kaplan-Meier curves were used to evaluate the prognostic value of levels of FNDC1 in BC. Furthermore, the biological functions of FNDC1 were investigated in two breast cancer cell lines. The present study assessed the effect of silencing FNDC1 on the proliferation, migration, invasion and epithelial-to-mesenchymal (EMT) transition of breast cancer cells.

\section{Materials and methods}

Cell culture and chemicals. Human BC cell lines (MCF-7, MDA-MB-468, LCC9 and T-47D) and the normal human breast MCF-10A cell line were purchased from the Cell Bank 
of Type Culture Collection of Chinese Academy of Sciences. All cells were maintained in RPMI-1640 medium (Gibco; Thermo Fisher Scientific, Inc.), supplemented with $10 \%$ FBS (Gibco; Thermo Fisher Scientific, Inc.) and 1\% penicillin and streptomycin (Sigma-Aldrich; Merck KGaA) in a humidified atmosphere of $5 \% \mathrm{CO}_{2}$ at $37^{\circ} \mathrm{C}$. All chemicals were purchased from Sigma-Aldrich (Merck KGaA).

Cell transfection. The lentivirus plasmids containing the short hairpin RNA (shRNA) against FNDC1 (shFNDC1) or a negative control (shNC) were synthesized by Suzhou GenePharma Co., Ltd. shRNAs were subcloned into pRNA-H1.1 (Thermo Fisher Scientific, Inc.). The plasmids were transfected into 293 T cells using Lipofectamine ${ }^{\circledR} 2000$ (Thermo Fisher Scientific, Inc.) according to the manufacturer's guide. Briefly, $24 \mathrm{~h}$ prior to the transfection, $2.5 \times 10^{6}$ cells were seeded into a $10 \mathrm{~cm}$ dish until confluency of $50-70 \%$ the next day. A total of $4 \mu \mathrm{g}$ lentiviral plasmid, $4 \mu \mathrm{g}$ each 3rd generation viral packaging vectors (pMDL, pRSV and pVSV-G) and $20 \mu 1$ Lipofectamine 2000 in $600 \mu \mathrm{l}$ serum-free DMEM was incubated for 15-20 min at room temperature and added into the cells. A total of $72 \mathrm{~h}$ later, the supernatant was collected. Prior to the viral transduction, cells were harvested upon reaching $70-80 \%$ confluence. Lentiviral vectors were added at an MOI of 50 into the plate. The medium was replaced with fresh medium $24 \mathrm{~h}$ after transfection. Then, cells were treated with puromycin $(2 \mu \mathrm{g} / \mathrm{ml})$ for 3 days and the transfection efficiency was evaluated by western blotting.

Cell viability assay. Cell viability was assayed by the Cell Counting Kit-8 (CCK-8) (Beyotime Institute of Biotechnology), according to the manufacturer's protocol. In brief, the cells were seeded onto 96 -well plates at the density of $5 \times 10^{3}$ cells/well. After infections for different times (24, 48, 72 and $96 \mathrm{~h}$ ), $10 \mu \mathrm{l} \mathrm{CCK}-8$ solution was added to each well and incubated for another $2 \mathrm{~h}$. The absorbance was measured at $450 \mathrm{~nm}$ by a microplate reader Elx808 (BioTek Instruments, Inc.).

Colony formation assay. Following transfection for $24 \mathrm{~h}$, the cells were seeded into the 6-well plates at a density of 500 cells/well in triplicate. After culturing for two weeks, the cells were washed with PBS, fixed with methanol for $0.5 \mathrm{~h}$ at room temperature and stained with crystal violet for $2 \mathrm{~h}$ at room temperature $(0.1 \%$; Beyotime Institute of Biotechnology). The colonies were visualized and counted under a CKX31 inverted light microscope at x100 magnification (Olympus Corporation).

Wound healing assay. A wound healing assay was performed with minor modifications to a previously reported method (9). As serum-free medium caused excessive apoptosis and cell detachment, 10\% FBS-supplemented medium was used $(9,10)$. In brief, cells were seeded onto 6-well plates in $10 \%$ FBS medium until they reached $80-90 \%$ confluency. The monolayers were scratched using $200 \mu \mathrm{l}$ sterile pipette tips, and the cells were washed with PBS three times to remove the debris and $10 \%$ FBS-supplemented fresh medium was added. A total of $24 \mathrm{~h}$ later, images were captured under a CKX31 inverted light microscope at x100 magnification (Olympus Corporation).
Invasion assay. For the cell invasion assay, $3 \times 10^{4}$ cells were seeded into the upper chamber of Transwell assay inserts (8 $\mu \mathrm{m}$; Corning Inc.). The Transwell chamber was precoated with Matrigel (Thermo Fisher Scientific, Inc.) before cell seeding. The chambers were inserted into a 24-well plate. The upper chambers were filled with $200 \mu \mathrm{l}$ serum-free medium (RPMI-1640; Gibco; Thermo Fisher Scientific, Inc.), while the bottom chambers were filled with $500 \mu$ l complete medium (RPMI-1640; Gibco; Thermo Fisher Scientific, Inc.). After incubation at $37^{\circ} \mathrm{C}$ for $24 \mathrm{~h}$, the cells were fixed at room temperature in $4 \%$ paraformaldehyde (Beyotime Institute of Biotechnology) for $10 \mathrm{~min}$ and stained at room temperature with $0.1 \%$ crystal violet (Beyotime Institute of Biotechnology) for $10 \mathrm{~min}$. Nonmigrating cells in the upper chambers were wiped off. The migrated cells were counted in three randomly selected fields and photographed at a X100 magnification with a CKX31 inverted light microscope at x100 magnification (Olympus Corporation).

Western blot analysis. The total cellular proteins were obtained using RIPA lysis buffer (Beyotime Institute of Biotechnology), supplemented with a proteinase and phosphatase inhibitor cocktail (Sigma-Aldrich; Merck KGaA). The protein concentration was assayed by Bradford kit (Beyotime Institute of Biotechnology) according to the manufacturer's guide. Twenty micrograms of total protein were loaded onto a $12 \%$ SDS-PAGE and then transferred onto a PVDF membrane (EMD Millipore). The membranes were blocked with skimmed milk for $1 \mathrm{~h}$ at room temperature, after which the membrane was incubated with primary antibodies against the following overnight at $4^{\circ} \mathrm{C}$ : FNDC1 (cat.no.PA5-56603; dilution, 1:1,000; Thermo Fisher Scientific, Inc.), GAPDH (cat. no. G9545; dilution, 1:10,000, Sigma-Aldrich; Merck KGaA), MMP-2 (cat. no. 40994; dilution, 1:1,000; Cell Signaling Technology, Inc.), MMP-9 (cat. no. 13667; dilution, 1:1,000; Cell Signaling Technology, Inc.), vimentin (cat. no. 5741; dilution, 1:1,000; Cell Signaling Technology, Inc.), N-cadherin (cat. no. 13116; dilution, 1:1,000; Cell Signaling Technology, Inc.), E-cadherin (cat. no. 14472; dilution, 1:1,000; Cell Signaling Technology, Inc.), p-ERK (cat. no. 4370; dilution, 1:1,000; Cell Signaling Technology, Inc.), ERK (cat. no. 4696; dilution, 1:1,000; Cell Signaling Technology, Inc.), p-JNK (cat. no. 9255; dilution, 1:1,000; Cell Signaling Technology, Inc.), JNK (cat. no. 9252; dilution, 1:1,000; Cell Signaling Technology, Inc.), p-p38 (cat. no. 4511; dilution, 1:1,000; Cell Signaling Technology, Inc.), p38 (cat. no. 8690; dilution, 1:1,000; Cell Signaling Technology, Inc.), p-Akt (cat. no. 4060; dilution, 1:1,000; Cell Signaling Technology, Inc.) and Akt (cat. no. 4685; dilution, 1:1,000; Cell Signaling Technology, Inc.). Subsequently, the membrane was washed three times with PBS and incubated with the corresponding horseradish peroxidase-conjugated secondary antibodies (both 1:2,000; anti-mouse secondary antibody; cat. no. 7076; and anti-rabbit secondary antibody; cat. no., 7074), which were obtained from Cell Signaling Technology, Inc. at room temperature for $1 \mathrm{~h}$. The membrane was visualized using an ECL Prime Western Blotting kit (cat. no. 21342; Beyotime Institute of Biotechnology). All western blots were repeated three times. Band intensities were quantified using NIH ImageJ (version 2.0; imgaej.niv. gov/). 
A

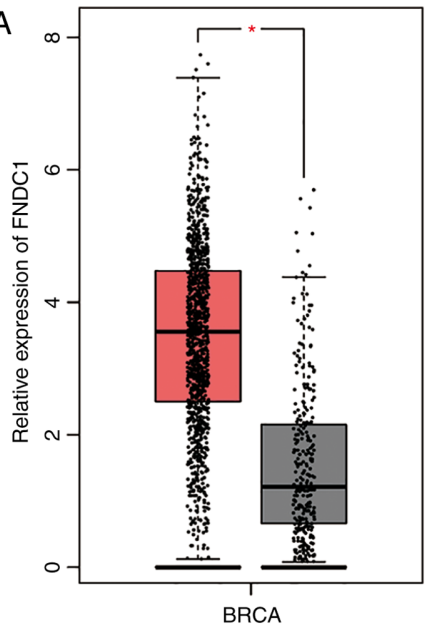

(num $(\mathrm{T})=1085 ; \operatorname{num}(\mathrm{N})=291)$

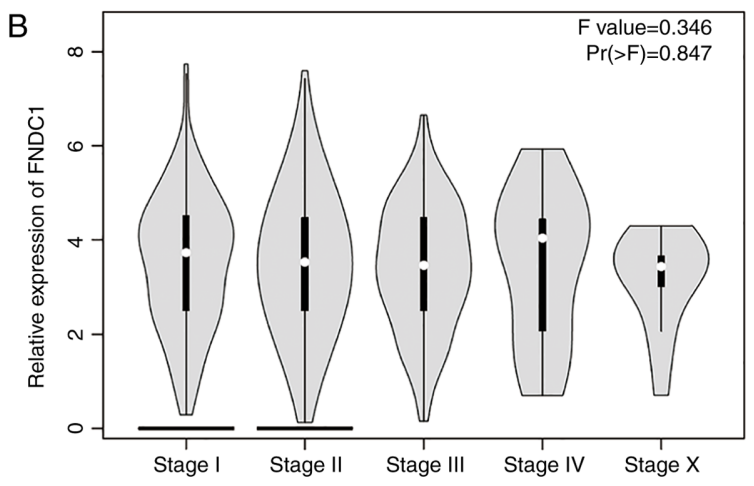

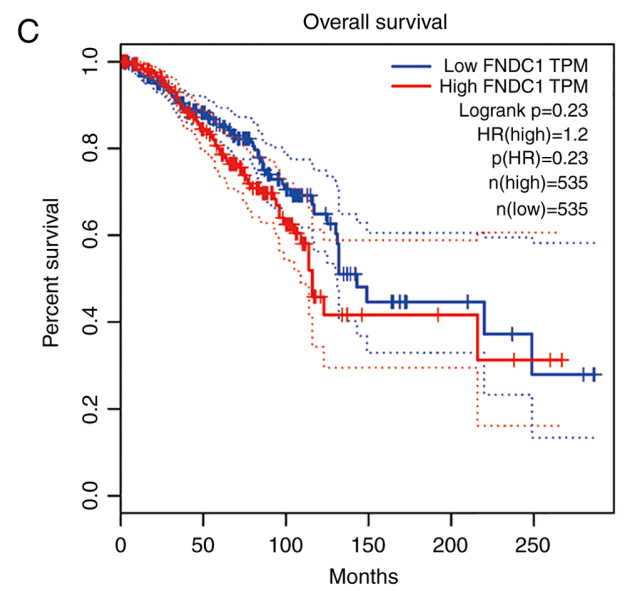

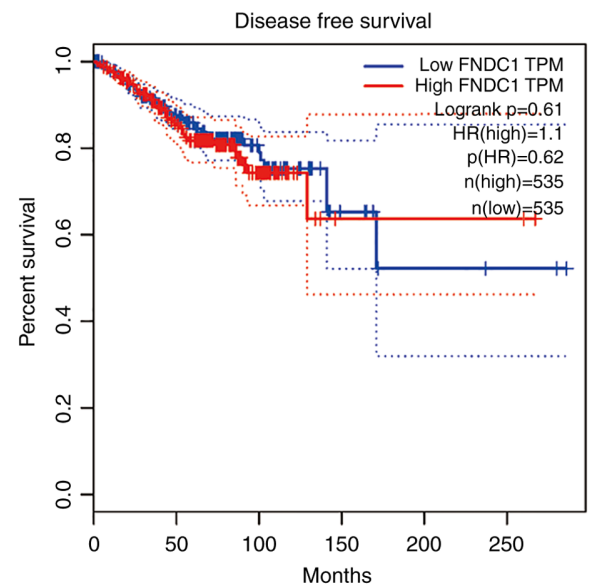

Figure 1. FNDC1 is upregulated in BC tissues. (A) Expression of FNDC1 in BC and corresponding normal tissues in TCGA database. (B) Expression of FNDC1 among the different stages of BC. (C) OS and DFS of patients with BC and the expression levels of FNDC1 in TCGA database. ${ }^{*} \mathrm{P}<0.05$. FNDC1, fibronectin type III domain-containing protein 1; BC, breast cancer; TCGA, The Cancer Genome Atlas; OS, overall survival; DFS, disease-free survival.

Data mining. The bioinformatics data for FNDC1 expression in BC were retrieved from The Cancer Genome Atlas (TCGA) (portal.gdc.cancer.gov) and the Genotype-Tissue Expression (GETx) database (gtexportal.org/home/) using the Gene Expression Profiling Interactive Analysis (GEPIA) tool ver.2002 (gepia.cancer-pku.cn). The associations of FNDC1 expression with overall survival (OS) and disease-free survival (DFS) were analyzed by data mining in the Kaplan-Meier plotter (kmplot.com). The median FNDC1 expression was applied as the cut-off. Hazard ratios with a $95 \%$ confidence interval (CI) and log-rank P-values were calculated.

Statistical analysis. Statistical analyses were performed with SPSS 12.0 (SPSS, Inc.). The data are expressed as the mean \pm standard deviation. The paired Student's t-test was used for comparisons between BC and normal tissues. In in vitro experiments, unpaired Student's t-test was used to compare the difference between two groups, and differences between multiple groups were analyzed using one-way analysis of variance followed by Tukey's post hoc test. Survival probability was calculated using the Kaplan-Meier method and the log-rank test was used to compare survival curves. $\mathrm{P}<0.05$ (two-tailed) was considered to indicate a statistically significant difference.

\section{Results}

Expression of FNDC1 in BC tissues. According to the data extracted from TCGA database, the expression of mRNA levels of FNDC1 was significantly upregulated in BC tissues when compared with normal tissues that were collected from the same patients (Student's t-test; Fig. 1A), although there was no significant difference in expression of FNDC1 among the different BC stages (Fig. 1B). The OS curve, which was constructed using the Kaplan-Meier method from TCGA database, demonstrated that there is no significant difference between the $\mathrm{BC}$ patients with high or low expression of FNDC1 (after dividing the patients' survival times by the median time, with a follow-up threshold of 250 months; $\mathrm{P}=0.23$; log-rank test; Fig. 1C). Furthermore, DFS curves did not show an association between the survival of patients with $\mathrm{BC}$ and the expression of FNDC1 (after dividing the patients' survival time by the median time, with a follow-up threshold of 250 months; $\mathrm{P}=0.61$; log-rank test; Fig. 1C). These findings unveil the complexity of FNDC1 in the tumorigenesis of BC.

Downregulation of FNDC1 inhibits the proliferation, colony formation, migration, invasion and EMT process of $B C$ cells. The biological functions of FNDC1 in BC cells were 
A

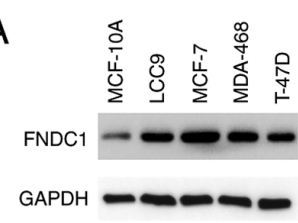

B

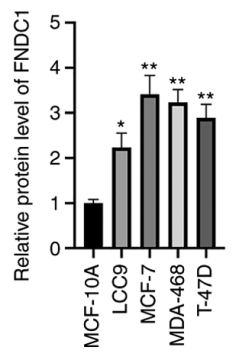

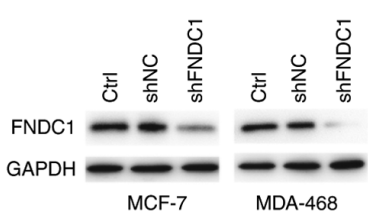
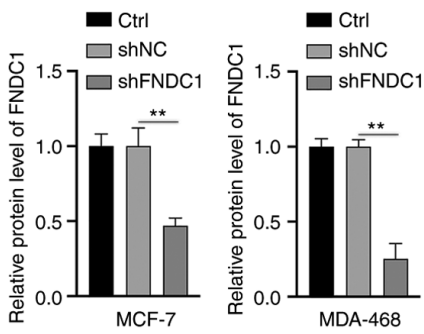
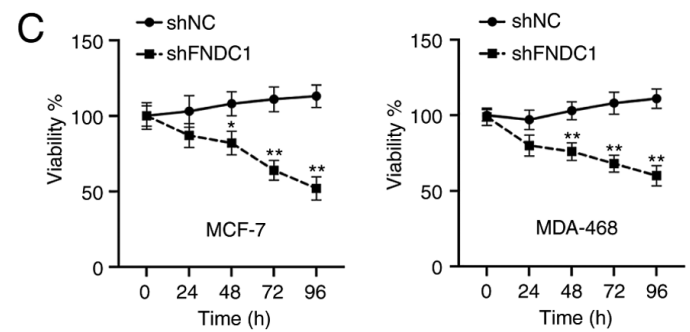

D
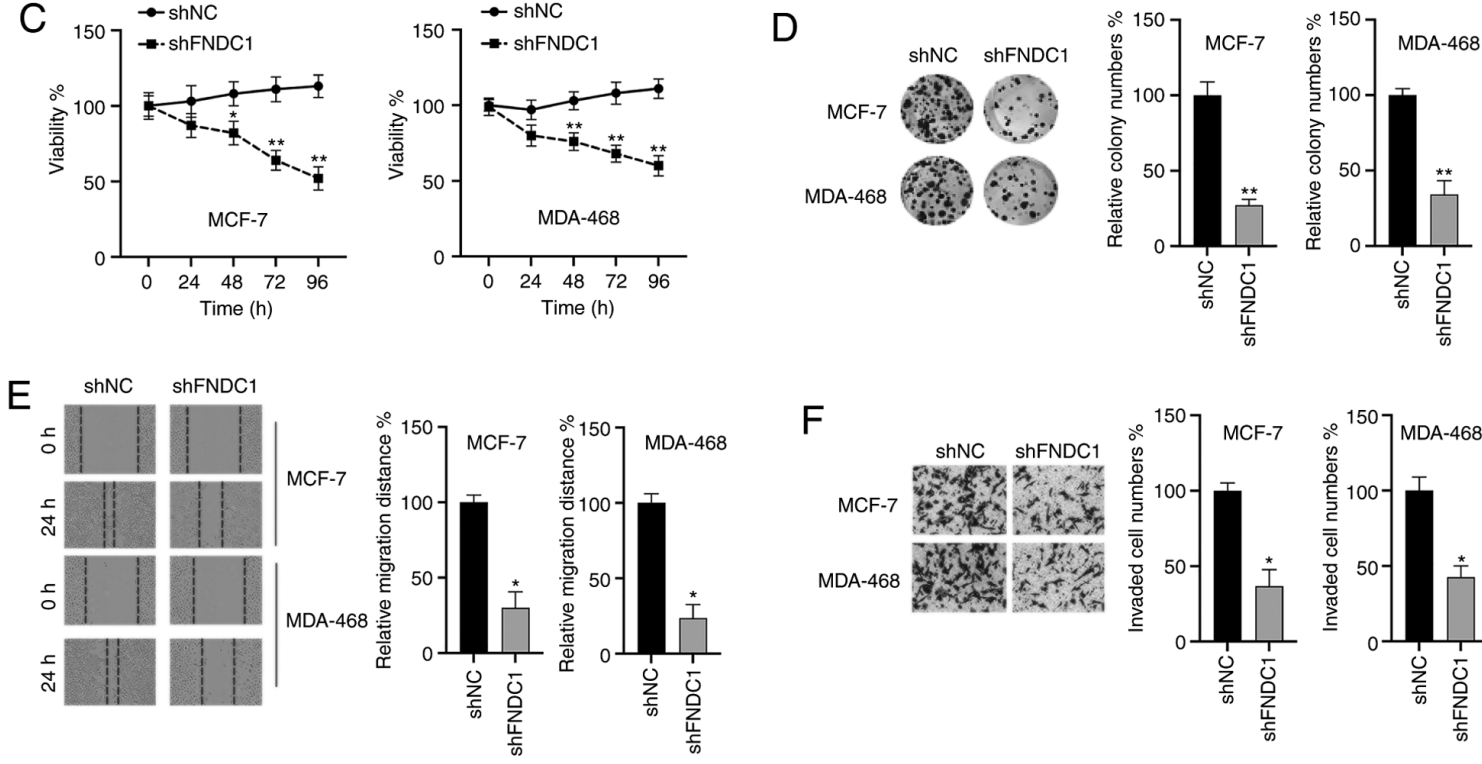

$\mathrm{F}$
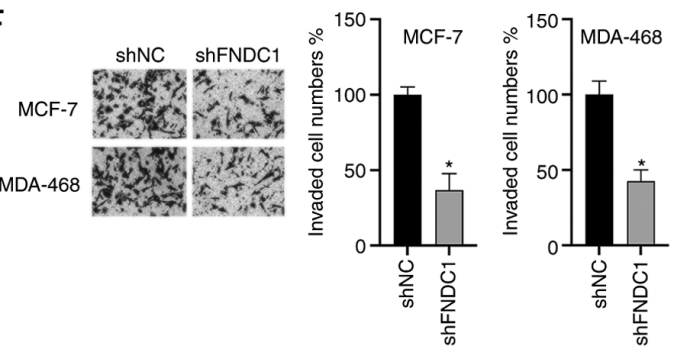

G
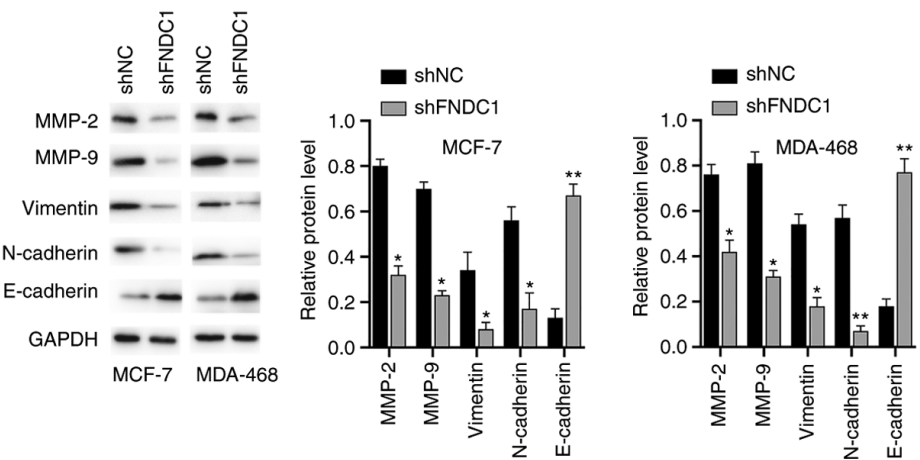

Figure 2. The silencing of FNDC1 inhibits the tumorigenesis of BC. (A) Expression of FNDC1 in normal breast cell and BC cells were assayed by western blotting. (B) BC cells were transfected as indicated for $24 \mathrm{~h}$, after which the protein levels of FNDC1 were measured by western blotting. (C) BC cells were transfected as indicated, after which the cell viability was measured by a Cell Counting kit-8 assay at different time points. (D) BC cells were transfected as indicated, after which a colony formation assay was performed. (E) BC cells were transfected as indicated, after which a wound healing assay was conducted to measure the migration ability. (F) BC cells were transfected as indicated, after which a Transwell assay was performed to measure the invasive ability. (G) BC cells were transfected as indicated for $24 \mathrm{~h}$, after which the expression levels of the indicated proteins were measured by western blotting. The data are presented as the mean \pm standard deviation. The experiments were performed at least three times. ${ }^{*} \mathrm{P}<0.05 ;{ }^{* * *} \mathrm{P}<0.01$. FNDC1, fibronectin type III domain-containing protein 1; $\mathrm{BC}$, breast cancer; sh, short hairpin RNA; $\mathrm{NC}$, negative control; $\mathrm{Ctrl}$, control.

investigated. The protein levels of FNDC1 in normal human breast MCF-10A cells and four breast cancer LCC9, MCF-7, MDA-468 and T-47D cell lines were assayed. As shown in Fig. 2A, the expression of FNDC1 was highest in MCF-7 and MDA-468 cells; therefore, these two cell lines were selected for the subsequent studies. Subsequently, the expression of FNDC1 was successfully silenced in two BC cell lines (MCF-7 and MDA-MB-468; Fig. 2B). A CCK-8 assay demonstrated that the silencing of FNDC1 significantly inhibited the viability of $\mathrm{BC}$ cells, compared with to the control group (Fig. 2C). Colony formation assays indicated that the colony numbers of $\mathrm{BC}$ cells were markedly decreased following knockdown of FNDC1 (Fig. 2D). Wound healing and Transwell invasion assays demonstrated that the silencing of FNDC1 significantly repressed the migration and invasion of BC cells (Fig. 2E and F). To reveal the molecular mechanisms, western blot analysis was performed. It was revealed that the silencing of FNDC1 led to the downregulation of MMP-2/9, 

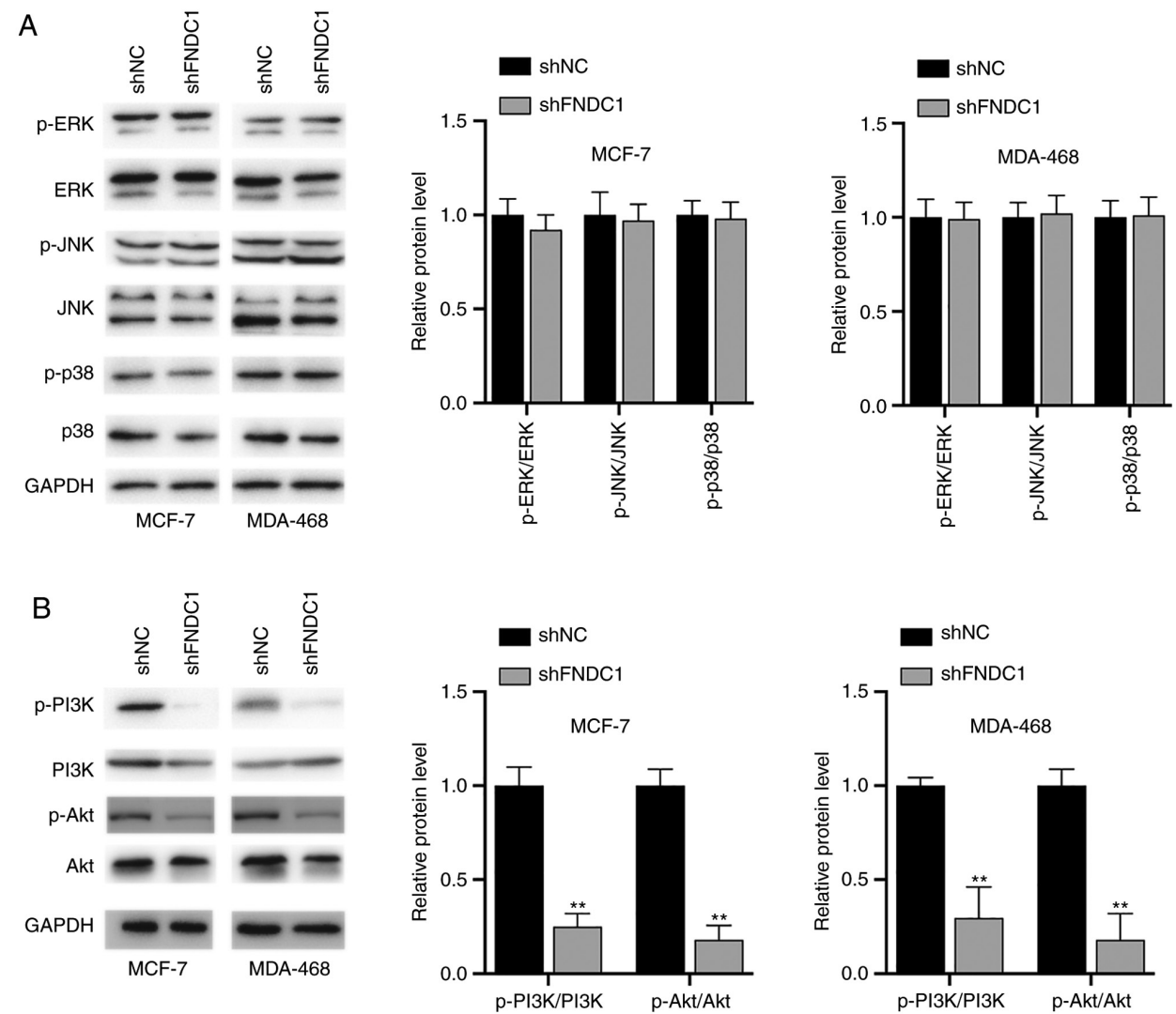

Figure 3. The silencing of FNDC1 leads to inhibition of the PI3K/Akt signaling pathway. (A and B) BC cells were transfected as indicated for $24 \mathrm{~h}$, after which the expression levels of the indicated proteins were measured by western blotting. The data are presented as the mean \pm standard deviation. The experiments were performed at least three times. ${ }^{* *} \mathrm{P}<0.01$. FNDC1, fibronectin type III domain-containing protein 1 ; sh, short hairpin RNA; NC, negative control.

vimentin and $\mathrm{N}$-cadherin and the upregulation of E-cadherin in BC cells (Fig. 2G). Taken together, these results suggested that the silencing of FNDC1 inhibits the tumorigenesis of BC cells.

Knockdown of FNDCl led to the inhibition of the PI3K/Akt signaling pathway. Whether FNDC1 has any effects on signaling pathways was investigated. To begin with, the status of the MAPK signaling pathways was investigated. It was demonstrated that ERK, JNK and p38 were not affected following the silencing of FNDC1 in BC cells (Fig. 3A). Subsequently, the PI3K/Akt signaling pathway was investigated. It was found that the phosphorylation levels of PI3K and Akt were decreased following the silencing of FNDC1 (Fig. 3B). Therefore, the silencing of FNDC1 led to the inhibition of PI3K.

Inactivation of the PI3K/Akt signaling pathway is essential for the effects of the silencing of FNDCl on BC cells. To verify whether the PI3K/Akt signaling pathway is required for the oncogenic effects of FNDC1, BC cells were transfected with shFNDC1 and then the cells were incubated with the Akt activator SC79 $24 \mathrm{~h}$ after transfection. It was revealed that SC79 reactivated the Akt signaling pathway following the silencing of FNDC1 in the two BC cell lines, as indicated by increased p-Akt/Akt ratios (Fig. 4A). In addition, it was found that SC79 reversed the effects of the silencing of FNDC1 on cell viability (Fig. 4B), migration (Fig. 4C) and invasion (Fig. 4D). Furthermore, the effects of the silencing of FNDC1 on MMPs and EMT markers were also reversed by the treatment of SC97 in $\mathrm{BC}$ cells (Fig. 4E). Taken together, these results suggested that the effects of the silencing of FNDC1 on the tumorigenesis of $\mathrm{BC}$ cells relied on the inactivation of the PI3K/Akt signaling pathway.

\section{Discussion}

The role of FNDC1 has been investigated in various cancer types, which demonstrated that it may be an oncogene. For instance, the overexpression of FNDC1 was found to be correlated with a poor prognosis in patients with gastric cancer (11). An upregulation of FNDC1 has also been revealed in head and neck squamous cell carcinoma and colorectal cancer $(12,13)$. However, there have been few studies regarding the roles of FNDC1 in the progression of BC. Therefore, the aim of the present study was to investigate the roles of FNDC1 in the tumorigenesis of $\mathrm{BC}$ cells via loss-of-function experiments. The underlying molecular mechanisms were also investigated.

In line with the results of previous studies, TCGA database analyses demonstrated that FNDC1 was upregulated in $\mathrm{BC}$ tissues. However, Kaplan-Meier analysis showed that no significant difference in OS time between the $\mathrm{BC}$ patients with high or low expression of FNDC1. Furthermore, the expression of FNDC1 was not associated with DFS in patients with BC. By contrast, previous studies have suggested that the expression of FNDC1 was associated with prognosis in gastric cancer $(11,14)$. These results suggested that the role of FNDC1 in tumors is complicated. Dysregulated proliferation is one of 
A
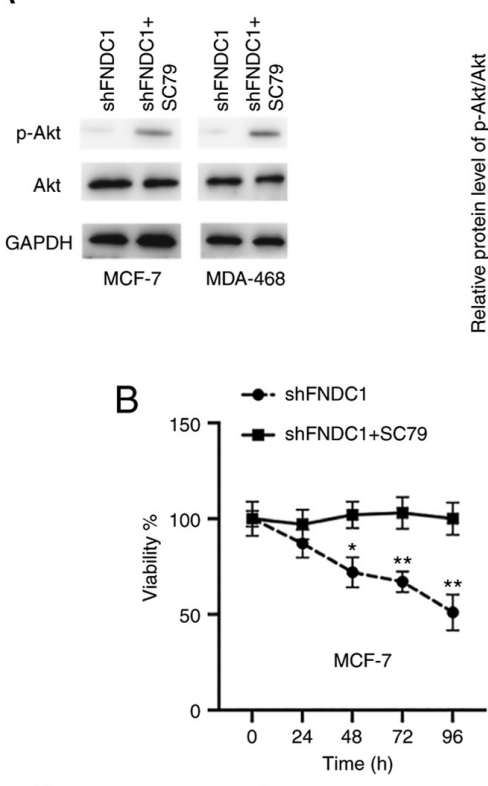

C
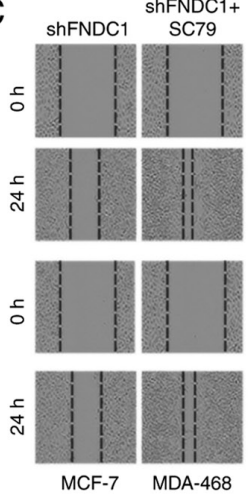

- shFNDC1

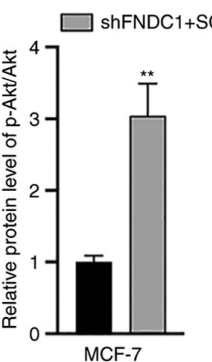

shFNDC1

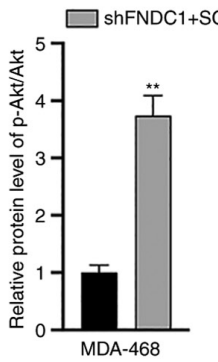

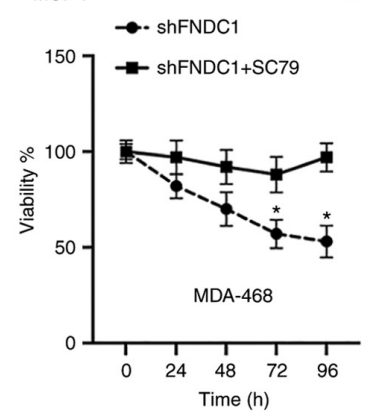
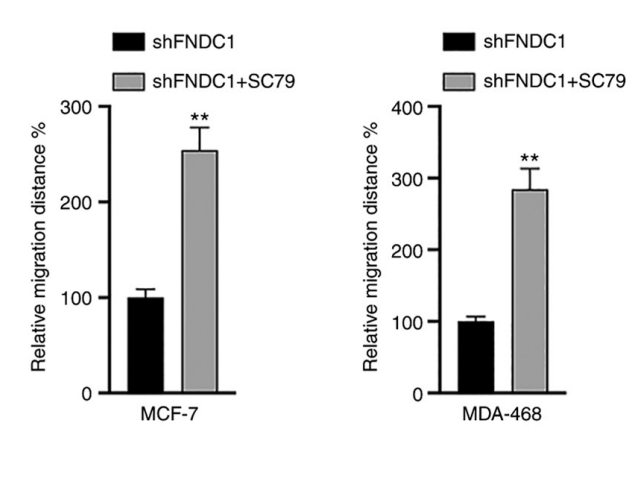

D
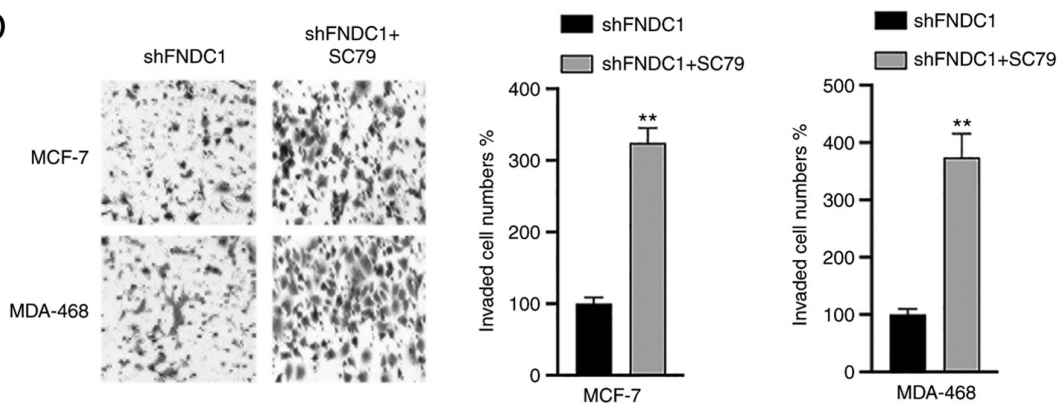

E
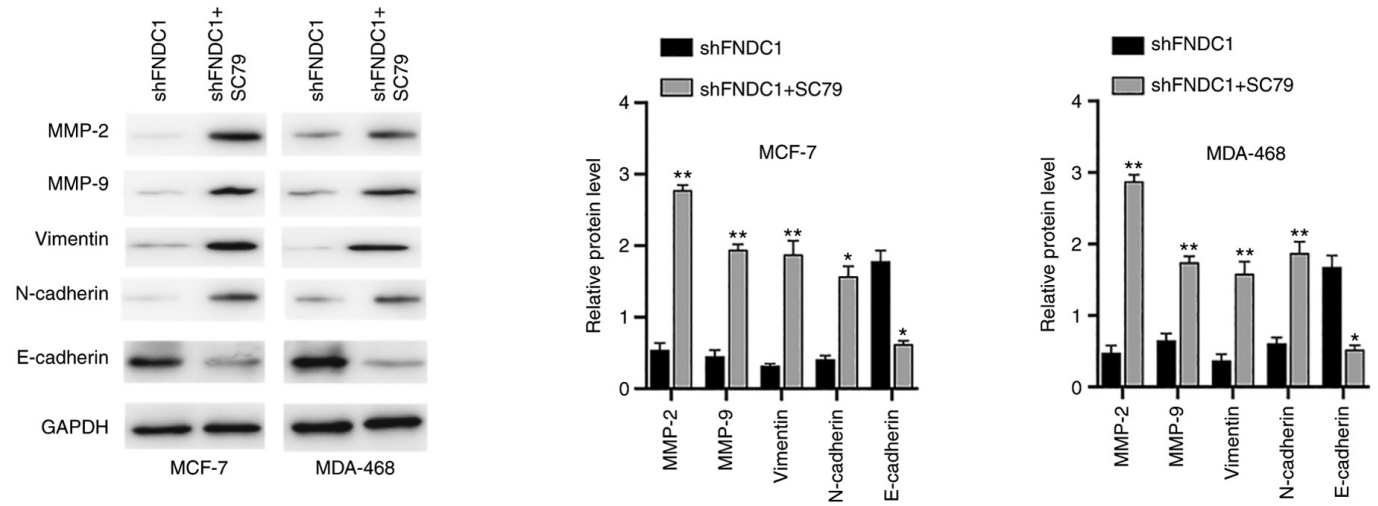

Figure 4. Activation of PI3K/Akt reverses the effects of the silencing of FNDC1 on BC cells. (A) BC cells were transfected with shFNDC1, and the cells were treated with or without SC79 for $24 \mathrm{~h}$, after which the indicated proteins were measured by western blotting. (B) BC cells were transfected with shFNDC1, and the cells were treated with or without SC79 for the indicated time. BC cells were transfected with shFNDC1, and the cells were treated with or without SC79 for $24 \mathrm{~h}$. (C) Migration was measured. (D) Invasion was measured. (E) The indicated proteins were measured. The data are presented as the mean \pm standard deviation. The experiments were performed at least three times. ${ }^{*} \mathrm{P}<0.05 ;{ }^{* *} \mathrm{P}<0.01$. FNDC1, fibronectin type III domain-containing protein 1 ; BC, breast cancer; sh, short hairpin RNA. 
the key hallmarks of cancer (15). In the present study, it was revealed that the knockdown of FNDC1 repressed the viability of BC cells. This finding is in line with that of a previous study, which reported that the downregulation of FNDC1 decreased the viability of prostate cancer cells (8). Additionally, the knockdown of FNDC1 may suppress the growth of gastric cancer cells (11). However, the roles of FNDC1 in the growth of $\mathrm{BC}$ cells remains unclear.

As another hallmark of cancer, metastasis is a leading cause of cancer-associated mortality (15). During metastasis, the surrounding tissues will be invaded by tumor cells, which will further intravasate into the lymph nodes and eventually migrate to distant organs (16). It is well documented that the extracellular matrix (ECM) serves essential roles in the process of metastasis (17). FN1 is an important ECM protein that has been revealed to enhance the invasiveness of various cancer cells, including gastric cancer cells, oral squamous cell carcinoma and ovarian cancer (18-20). Since FNDC1 contains a major component of the structural domain of FN1, this indicates that FNDC1 may also serve a critical role in metastasis (5). In the present study, it was demonstrated found that the silencing of FNDC1 inhibited the migration and invasion of BC cells, which is in line with the results of previous studies $(11,21)$. Investigation of mechanisms revealed that the silencing of FNDC1 decreased the expression of MMPs, which are a group of zinc-dependent endopeptidases (22). MMP-2 and MMP-9 participate in the process of cell invasion and metastasis via regulation of the degradation of the ECM (23). A decrease in MMP-2/9 may repress the migration and metastasis of tumor cells (24). It was found that the silencing of FNDC1 led to the downregulation of MMP-2/9, which revealed the molecular mechanisms underlying the repression of migration and invasion caused by the silencing of FNDC1. It has been well documented that the EMT serves an essential role in the progression of various cancer types, including BC (25). EMT accounts for the change in various cell phenotypes that are involved in the progression of tumors (26). The results of the present study demonstrated that the silencing of FNDC1 led to the downregulation of proteins, including vimentin and $\mathrm{N}$-cadherin and to the upregulation of E-cadherin. These data suggested that the EMT process was inhibited by the downregulation of FNDC1. The findings of the present study are in accordance with those of a previous study, which also found that the silencing of FNDC1 blocked the EMT process in gastric cancer cells (11).

The PI3K/Akt signaling pathway has been reported to participate in cancer cell activities, including metabolism, proliferation, invasion and metastasis (27). The constitutive activation of Akt has been found to contribute toward the initiation and development of different cancer types, including BC (28). Targeting PI3K/Akt may be a potential strategy for treatment and for overcoming drug resistance in BC (29). Until now, whether FNDC1 may modulate the PI3K/Akt signaling pathway has remained elusive. The present study found that the downregulation of FNDC1 led to the inactivation of the PI3K/Akt pathway. In addition, SC79 reversed the inhibitory effects of the silencing of FNDC1 on tumorigenesis in BC cells. To the best of our knowledge, the present study was the first to indicate that the downregulation of FNDC1 leads to the inhibition of the PI3K/Akt pathway. Notably, a previous study demonstrated that the Akt signaling pathway may regulate the expression of FNDC1 in bone marrow mesenchymal stem cells (30). Therefore, there may be a correlation between the PI3K/Akt pathway and FNDC1; therefore, more studies are required. There are certain limitations to the present study. To begin with, the expression of FNDC1 was not assayed in patients with $\mathrm{BC}$ and the levels of FNDC1 in BC tissues should be measured in the future. Additionally, the effects of FNDC1 on the tumorigenesis of BC were not studied in a xenograft mouse model, which may be interesting if performed in the future.

In summary, the present study revealed that FNDC1 expression is upregulated in $\mathrm{BC}$. The silencing of FNDC1 inhibited the proliferation, migration, invasion and EMT of BC cells by modulating the PI3K/Akt signaling pathway. Therefore, FNDC1 may be a potential therapeutic target for the treatment of $\mathrm{BC}$.

\section{Acknowledgements}

Not applicable.

\section{Funding}

The present study was supported by the Medical Scientific Research Foundation of Zhejiang Province, China (grant no. 2019KY596).

\section{Availability of data and materials}

The datasets used and/or analyzed during the current study are available from the corresponding author on reasonable request.

\section{Authors' contributions}

CY and GS performed the experiments. CY and GS confirm the authenticity of all the raw data. GS performed the statistical analysis. BZ and CS performed the bioinformatics analysis. YH designed the study and drafted the manuscript. All authors read and approved the final manuscript.

\section{Ethics approval and consent to participate}

Not applicable.

\section{Patient consent for publication}

Not applicable.

\section{Competing interests}

The authors declare that they have no competing interests.

\section{References}

1. DeSantis CE, Ma J, Goding Sauer A, Newman LA and Jemal A: Breast cancer statistics, 2017, racial disparity in mortality by state. CA Cancer J Clin 67: 439-448, 2017.

2. Miller KD, Nogueira L, Mariotto AB, Rowland JH, Yabroff KR, Alfano CM, Jemal A, Kramer JL and Siegel RL: Cancer treatment and survivorship statistics, 2019. CA Cancer J Clin 69: 363-385, 2019. 
3. Khosravi-Shahi P, Cabezon-Gutierrez L and Custodio-Cabello S Metastatic triple negative breast cancer: Optimizing treatment options, new and emerging targeted therapies. Asia Pac J Clin Oncol 14: 32-39, 2018

4. Pankov R and Yamada KM: Fibronectin at a glance. J Cell Sci 115: 3861-3863, 2002

5. Albrecht M, Renneberg H, Wennemuth G, Möschler O, Janssen M, Aumüller G and Konrad L: Fibronectin in human prostatic cells in vivo and in vitro: Expression, distribution, and pathological significance. Histochem Cell Biol 112: 51-61, 1999.

6. Fernandez-Garcia B, Eiro N, Marin L, González-Reyes S, González LO, Lamelas ML and Vizoso FJ: Expression and prognostic significance of fibronectin and matrix metalloproteases in breast cancer metastasis. Histopathology 64: 512-522, 2014.

7. Anderegg U, Breitschwerdt K, Kohler MJ, Sticherling M Haustein UF, Simon JC and Saalbach A: MEL4B3, a nove mRNA is induced in skin tumors and regulated by TGF-beta and pro-inflammatory cytokines. Exp Dermatol 14: 709-718, 2005.

8. Das DK, Naidoo M, Ilboudo A, Park JY, Ali T, Krampis K, Robinson BD, Osborne JR and Ogunwobi OO: miR-1207-3p regulates the androgen receptor in prostate cancer via FNDC1/fibronectin. Exp Cell Res 348: 190-200, 2016.

9. Valster A, Tran NL, Nakada M, Berens ME, Chan AY and Symons M: Cell migration and invasion assays. Methods 37: 208-215, 2015.

10. Pijuan J, Barcelo C, Moreno DF, Maiques O, Sisó P, Marti RM, Macià $\mathrm{A}$ and Panosa $\mathrm{A}$ : In vitro cell migration, invasion, and adhesion assays: From cell imaging to data analysis. Front Cell Dev Biol 7: 107, 2019

11. Liu YP,Chen WD,Li WN andZhang M: Overexpression of FNDC1 relates to poor prognosis and its knockdown impairs cell invasion and migration in gastric cancer. Technol Cancer Res Treat: Jan 1, 2019 (Epub ahead of print). doi: 10.1177/1533033819869928.

12. Wuensch T, Wizenty J, Quint J, Spitz W, Bosma M, Becker O, Adler A, Veltzke-Schlieker W, Stockmann M, Weiss S, et al: Expression analysis of fibronectin type III domain-containing (FNDC) genes in inflammatory bowel disease and colorecta cancer. Gastroenterol Res Pract 2019: 3784172, 2019.

13. Reddy RB, Khora SS and Suresh A: Molecular prognosticators in clinically and pathologically distinct cohorts of head and neck squamous cell carcinoma-A meta-analysis approach. PLoS One 14: e0218989, 2019.

14. Zhong M, Zhang Y, Yuan F, Peng Y, Wu J, Yuan J, Zhu W and Zhang Y: High FNDC1 expression correlates with poor prognosis in gastric cancer. Exp Ther Med 16: 3847-3854, 2018.

15. Hanahan D and Weinberg RA: Hallmarks of cancer: The next generation. Cell 144: 646-674, 2011.

16. Clark AG and Vignjevic DM: Modes of cancer cell invasion and the role of the microenvironment. Curr Opin Cell Biol 36: 13-22, 2015.

17. Eble JA and Niland S: The extracellular matrix in tumor progression and metastasis. Clin Exp Metastasis 36: 171-198, 2019.
18. Hao S, Lv J, Yang Q, Wang A, Li Z, Guo Y and Zhang G: Identification of key genes and circular RNAs in human gastric cancer. Med Sci Monit 25: 2488-2504, 2019.

19. Chen Z, Tao Q, Qiao B and Zhang L: Silencing of LINC01116 suppresses the development of oral squamous cell carcinoma by up-regulating microRNA-136 to inhibit FN1. Cancer Manag Res 11: 6043-6059, 2019.

20. Liang H, Yu M, Yang R, Zhang L, Zhang L, Zhu D, Luo H, Hong Y, Yu T, Sun J, et al: A PTAL-miR-101-FN1 axis promotes EMT and invasion-metastasis in serous ovarian cancer. Mol Ther Oncolytics 16: 53-62, 2019

21. Ren J, Niu G, Wang X, Song T, Hu Z and Ke C: Overexpression of FNDC1 in gastric cancer and its prognostic significance. J Cancer 9: 4586-4595, 2018.

22. Klein T and Bischoff R: Physiology and pathophysiology of matrix metalloproteases. Amino Acids 41: 271-290, 2011.

23. Nemeth JA, Yousif R, Herzog M, Che M, Upadhyay J, Shekarriz B, Bhagat S, Mullins C, Fridman R and Cher ML: Matrix metalloproteinase activity, bone matrix turnover, and tumor cell proliferation in prostate cancer bone metastasis. J Natl Cancer Inst 94: 17-25, 2002.

24. Huang H: Matrix metalloproteinase-9 (MMP-9) as a cancer biomarker and MMP-9 biosensors: Recent advances. Sensors (Basel) 18: 3249, 2018

25. Kotiyal S and Bhattacharya S: Breast cancer stem cells, EMT and therapeutic targets. Biochem Biophys Res Commun 453: 112-116, 2014.

26. Vincent CT and Fuxe J: EMT, inflammation and metastasis. Semin Cancer Biol 47: 168-169, 2017.

27. Aoki M and Fujishita T: Oncogenic roles of the PI3K/AKT/mTOR axis. Curr Top Microbiol Immunol 407: 153-189, 2017.

28. Sharma VR, Gupta GK, Sharma AK, Batra N, Sharma DK, Joshi A and Sharma AK: PI3K/Akt/mTOR intracellular pathway and breast cancer: Factors, mechanism and regulation. Curr Pharm Des 23: 1633-1638, 2017.

29. Guerrero-Zotano A,MayerIA and ArteagaCL:PI3K/AKT/mTOR: Role in breast cancer progression, drug resistance, and treatment. Cancer Metastasis Rev 35: 515-524, 2016.

30. Xiao Y, Wei R, Yuan Z, Lan X, Kuang J, Hu D, Song Y and Luo J: Rutin suppresses FNDC1 expression in bone marrow mesenchymal stem cells to inhibit postmenopausal osteoporosis. Am J Transl Res 11: 6680-6690, 2019.

This work is licensed under a Creative Commons Attribution-NonCommercial-NoDerivatives 4.0 International (CC BY-NC-ND 4.0) License. 